\title{
Multisystem Langerhans cell histiocytosis with liver dysfunction as the first presentation: A case report
}

\author{
DIAN-GANG LIU ${ }^{1}$, YU-XIAN ZHANG ${ }^{2}$ and FEI LI ${ }^{1}$ \\ ${ }^{1}$ Department of General Surgery, Xuanwu Hospital; ${ }^{2}$ Liver Research Center, \\ Beijing Friendship Hospital, Capital Medical University, Beijing 100053, P.R. China
}

Received June 14, 2011; Accepted October 18, 2011

DOI: $10.3892 / \mathrm{ol} .2011 .462$

\begin{abstract}
Langerhans cell histiocytosis $(\mathrm{LCH})$ is a rare disease of unknown etiology characterized by oligoclonal proliferation of Langerhans cells. The diagnosis of $\mathrm{LCH}$ is complicated by the fact that it may involve multiple organ systems and its clinical presentation and course varies, ranging from an isolated to a multisystem disease. We report a 35-year-old male with LCH involving multiple systems, including the bones, lungs, spleen, liver and bile ducts, whose first clinical presentation was liver dysfunction. The patient was diagnosed following a skull biopsy that revealed infiltration of Langerhans cells. However, a liver biopsy revealed sclerosing cholangitis (SC) with no signs of Langerhans cell infiltration, and the clinical manifestations of the involved organs were atypical, leading to a delayed diagnosis. The patient was in partial remission following chemotherapy. In conclusion, findings of this case may aid our understanding of the pathophysiology of LCH and in improving its diagnosis and treatment.
\end{abstract}

\section{Introduction}

Langerhans cell histiocytosis ( $\mathrm{LCH}$ ) is characterized by the proliferation of abnormal dendritic antigen-presenting histiocytes. LCH may involve almost any organ. The clinical presentation of $\mathrm{LCH}$ therefore varies and is frequently misdiagnosed (1-3). We report a patient who was initially admitted to the liver department with sclerosing cholangitis (SC), but was eventually diagnosed with multisystem LCH.

\section{Case report}

A 35-year-old Chinese male was admitted to our hospital with a 2-year history of jaundice and pruritus. The patient's laboratory test results are shown in Table I. Magnetic resonance

Correspondence to: Dr Dian-Gang Liu, Department of General Surgery, Xuanwu Hospital, Capital Medical University, No. 45 Changchun Street, Xuanwu, Beijing 100053, P.R. China

E-mail: liudiangang01@gmail.com

Key words: Langerhans cell histiocytosis, sclerosing cholangitis, multiple myeloma cholangiopancreatography (MRCP) and the pathology of a liver biopsy supported the diagnosis of SC by a local hospital. The patient received treatment with ursodeoxycholic acid (UDCA) at a dose of $750 \mathrm{mg} / \mathrm{day}$, with no marked response. The patient had no history of smoking, alcohol consumption or blood transfusions, and no family history of similar symptoms. Physical examinations revealed mildly icteric sclera and skin, coarse breath sounds in the lungs and enlargement of the spleen accompanied by percussion pain. A $1.0 \times 1.0-\mathrm{cm}$ defect was detected in the patient's occipital bone, which had occasionally caused pain over the previous 8 months.

The laboratory examination showed a normal complete blood count, positive urine bilirubin and urobilinogen, a normal stool test and an erythrocyte sedimentation rate (ESR) of $26 \mathrm{~mm} / \mathrm{h}$. Liver function test results are shown in Table I. Viral hepatitis markers were negative. Immunoglobulin (IgG) was $1,920.0 \mathrm{mg} / \mathrm{dl}$. Antinuclear antibody (1:80) (normal <1:80) was positive. Anti-smooth muscle, anti-liver/kidney microsome, anti-soluble liver antigen and liver cytosol antigen type I antibody were all negative. Anti-mitochondrial antibody subtype-2, extractable nuclear antigen and double-stranded DNA (ds-DNA) were negative. $\alpha$-fetoprotein, carcinoembryonic antigen and CA19-9 concentrations were also normal. Body mass index was normal.

An X-ray examination showed multifocal osteolytic bone lesions, including the right occipital bone (Fig. 1A), left iliac bone, left neck of the femur (Fig. 1B) and the seventh posterior segment of the rib. Chest radiography (Fig. 1C) and computed tomography (CT) scans of the lungs (Fig. 1D) revealed marked reticular opacities and diffuse small cysts. CT showed multiple low-density lesions in the liver. The lesions were shown as hyperechoic areas on ultrasound examination, while isointense intensity areas on the T1-weighted image and low intensity areas without enhancement on the axial fatsuppressed T2-weighted image were visualized by magnetic resonance imaging (MRI) (Fig. 2A). This observation suggested the possibility of fat deposition on the liver. MRCP (Fig. 2B) was highly suggestive of SC, including segmental intrahepatic bile duct dilatation and stenosis, and proximal strictures of the right and left hepatic ducts. However, the common hepatic duct exhibited no stenosis or dilatation.

Fine-needle liver biopsy under ultrasound guidance and hematoxylin-eosin staining showed interstitial fibrosis and inflammatory infiltrates in the portal area, epithelial injury 
Table I. Comparison of figures at admission and following chemotherapy.

\begin{tabular}{lcccc}
\hline & 2 years ago & At admission & Following chemotherapy & Normal range \\
\hline ALT (U/l) & 134 & 110 & 52 & $0-40$ \\
AST (U/l) & 78 & 81 & 30 & $0-40$ \\
GGT (U/l) & 698 & 73 & 113 & $8-55$ \\
ALP (U/l) & 538 & 431 & 303 & $30-150$ \\
TBIL $(\mu \mathrm{mol} / \mathrm{l})$ & 17 & 67 & 34 & $3.42-17.1$ \\
DBIl $(\mu \mathrm{mol} / \mathrm{l})$ & 6.5 & 38.7 & 13 & $0-6.84$ \\
ESR $(\mathrm{mm} / \mathrm{h})$ & 35 & 26 & 10 & $0-20$ \\
Immunoglobin IgG $(\mathrm{mg} / \mathrm{dl})$ & 1890 & 1920 & 1550 & $723-1685$ \\
\hline
\end{tabular}

ALT, alanine aminotransferase; AST, aspartate aminotransferase; GGT, $\gamma$-glutamyl transferase; ALP, alkaline phosphatase; TBIL, total bilirubin; DBI1, direct bilirubin; ESR, erythrocyte sedimentation rate.

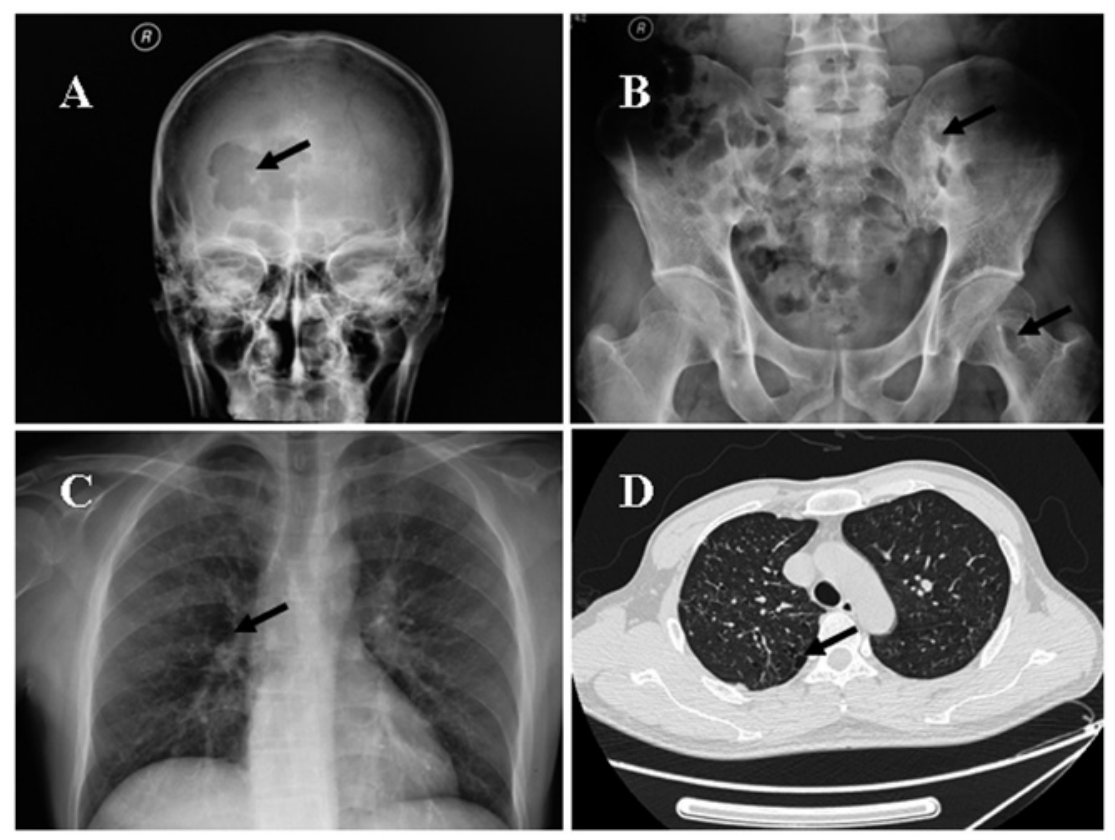

Figure 1. (A) Frontal radiographs of the skull showing extensive geographic lytic lesions of right occipital bone. (B) Ellipse lesions are present in the left neck of the femur and left iliac. (C) The chest radiograph shows marked reticular opacities and lytic lesions in the seventh posterior segment of the ribs. (D) High resolution $\mathrm{CT}$ of the chest (lung window) shows multiple cysts and nodules; multiple cysts have coalesced to form larger cysts of variable sizes and wall thickness.

and reactive hyperplasia of small bile ducts with cholestatic features, phagocyte aggregation and lipid granuloma formation (Fig. 2C and D), suggesting chronic intrahepatic cholestasis and moderate steatosis. However, eosinophils or macrophages were not found and immunohistochemical stains for CD1a and S-100 protein were negative. The occipital bone biopsy revealed bone granulomas consisting predominantly of macrophages, intermixed with eosinophils (Fig. 3A). The macrophages were strongly positive for antibodies to CD1a (Fig. 3B) and S100 (Fig. 3C) antigens. Immunohistochemical analysis showed that $10 \%$ of cells were positive for the proliferation marker Ki-67 (Fig. 3D). We also investigated the involvement of other organs. Bone marrow puncture showed bone reactive hyperplasia. Brain MRI revealed no abnormalities of the hypothalamic-pituitary region. No thyroid lesions were detected by ultrasonography, and gastroscopy and colonoscopy revealed no involvement of the gastrointestinal tract.

The patient was diagnosed with multisystem, high-risk organ LCH and was referred to the hematological department for specific treatment. A first course of chemotherapy (vinblastine $6 \mathrm{mg} / \mathrm{m}^{2}$ i.v. bolus; prednisone $40 \mathrm{mg} / \mathrm{m}^{2} /$ day orally, weekly reduction after week 4) was administered. Although the patient developed adverse effects of chemotherapy, such as bacterial infection, chemotherapy was continued and supportive treatment lead to bone pain being relieved, liver function improving and normalization of ESR (Table I).

\section{Discussion}

LCH often presents as a puzzling syndrome and is difficult to diagnose in adults. The incidence of LCH in adults may 


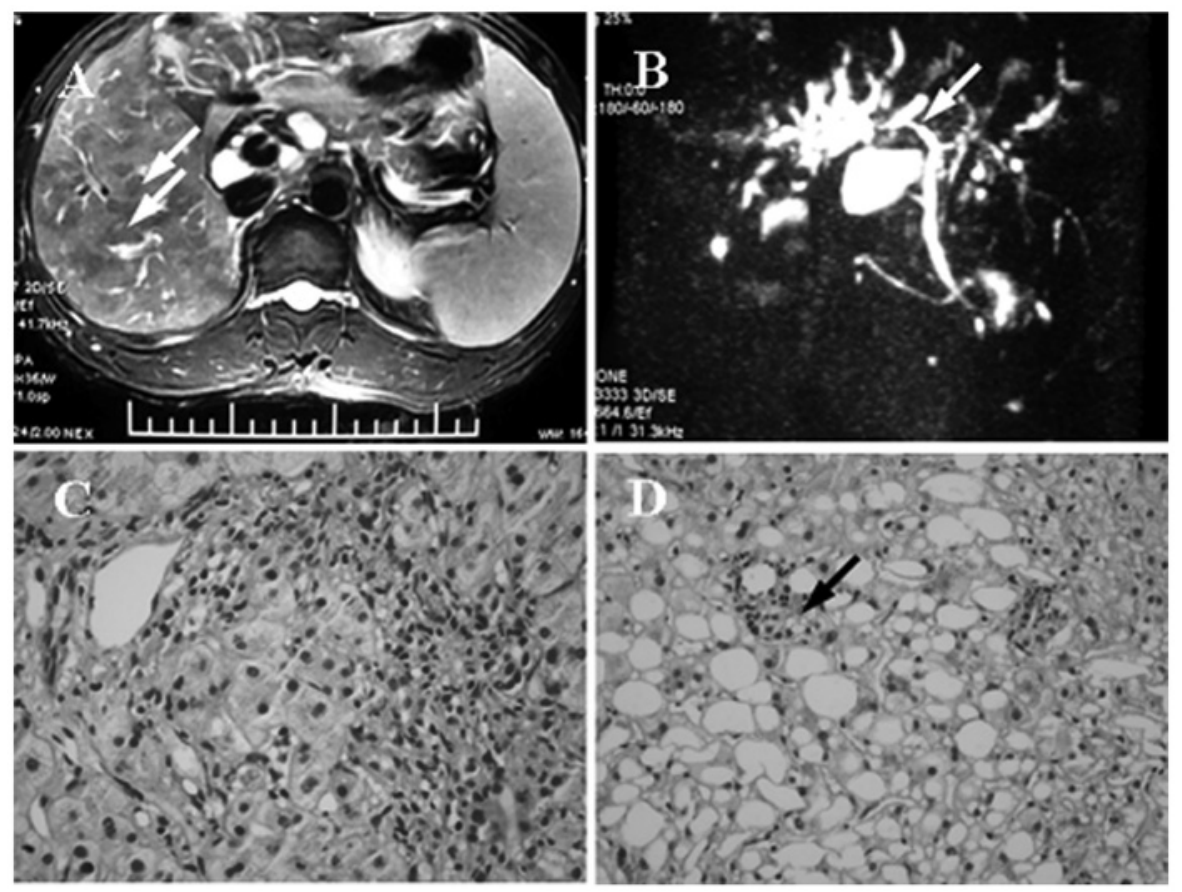

Figure 2. (A) Multifocal low density lesion in the liver, particularly the right liver, is shown. The lesions do not increase on the enhanced scan (white arrow). (B) MRCP suggests sclerosing cholangitis, including segmental intrahepatic bile duct dilatation and stenosis (white arrow), and proximal strictures of right and left hepatic ducts (white arrow). (C and D) Liver biopsy specimen is shown. (C) Hematoxylin and eosin staining (H\&E) showed interstitial fibrosis and inflammatory infiltrates in the portal area, epithelial injury and reactive hyperplasia of small bile ducts with cholestatic features (magnification, x60). (D) H\&E showed centrilobular zone macrovesicular steatosis and bilirubin pigment granules, phagocyte aggregation and lipid granuloma formation (black arrow) (magnification, $\mathrm{x} 40$ ).
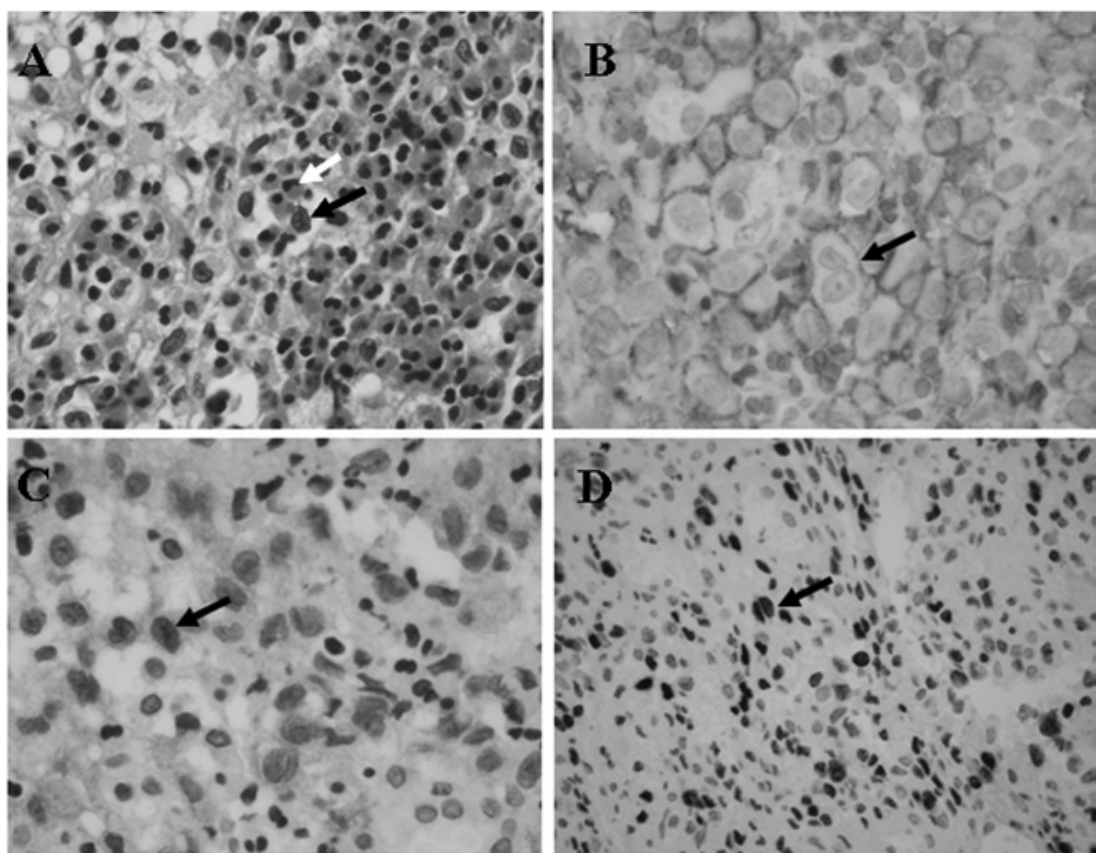

Figure 3. The occipital bone biopsy tissue is shown. (A) The image shows diffusely infiltrated areas destroyed by Langerhans cells (black arrow) and eosinophils intermixed (white arrow) (HE; magnification, x100). (B) Positive immunohistochemical staining for CD1a antigen (CD1a, x60), (C) S-100 protein (S-100, x60) and (D) Ki-67 (Ki-67, x60, 10\% of cells).

reach 1-2 cases per million and is thus significantly lower than that in children (4). The 35-year-old patient came to the liver department with liver dysfunction as the first symptom, and the patient's history and laboratory examination excluded the common causes of liver dysfunction. SC and multifocal osteolytic bone lesions made us consider the possibility of a single disease with multisystem involvement.

However, the immunohistochemical analysis of the liver biopsy yielded negative results for CD1a and S-100, possibly as the disease process is usually found around the major bile 
ducts, and a blind liver biopsy may therefore miss the $\mathrm{LCH}$ infiltration, and as the number of Langerhans cells varies in different lesions, with limited numbers observed in the liver, spleen, gastrointestinal or central nervous system tissues (5). Secondly, the liver biopsy and results of MRCP supported a diagnosis of SC. When LCH infiltrates the liver, it shows a propensity for the biliary ducts, leading to the development of cholestasis with an increase in GGT and ALP. The TB levels do not appear to vary in correlation to GGT or ALP. The pathological pattern ranges from mild cholestasis to more severe periportal infiltration with consequent hepatocellular injury in LCH. Bile duct involvement is capable of progressing to a SC-type image, fibrosis and eventually to liver failure. Although SC patients typically present with a cholestatic biochemical profile with 3-10 times the upper limit of serum ALP, this finding is neither specific nor mandatory (6). The serum levels increase with fluctuations due to choledocholithiasis or dominant stenoses with progression of the disease.

This progressive damage may continue, despite the regression of the $\mathrm{LCH}(6,7)$. Diagnostic imaging was able to visualize areas of $\mathrm{LCH}$ infiltration that may have been missed by biopsy. Imaging findings of liver involvement may vary depending on the progressive histological phase: proliferative, granulomatous, xanthomatous or fibrous phases (8). Diagnostic imaging results of this study have shown that the liver damage in this patient was in the xanthomatous stage.

Various treatment strategies have been employed in adult $\mathrm{LCH}$ patients, depending on the degree of organ involvement and the clinical course. Treatment of multisystem $\mathrm{LCH}$ normally benefits from systemic therapy, which usually reduces morbidity and mortality $(3,9)$. Our patient received an initial 6-week course of therapy (prednisone $40 \mathrm{mg} / \mathrm{m}^{2} /$ day orally, weekly reduction after week 4 , vinblastine $6 \mathrm{mg} / \mathrm{m}^{2}$ i.v. bolus, weekly) according to the Histiocyte Society Evaluation and Treatment Guidelines (10). Although the patient achieved partial remission following the first course of chemotherapy, the patient's prognosis remained uncertain. A number of studies have reported high mortality (30-50\%) when high-risk organs including the liver, spleen, lungs and hematopoietic system are involved, compared with the situation when these areas are not involved $(<10 \%)$. The 3 -year survival rate with liver involvement is $51.8 \%$, compared with that of $96.7 \%$ without liver involvement (11).

In conclusion, our case is a rare presentation of multisystem LCH with liver dysfunction as the first presentation in an adult. Particular attention should be paid to osteolytic skull lesions associated with liver dysfunction or pulmonary symptoms in order to gain a better understanding of the pathophysiology of $\mathrm{LCH}$ and improve diagnosis and treatment.

\section{References}

1. Yağci B, Varan A, Cağlar M, Söylemezoğlu F, Sungur A, Orhan D, Yalçin B, Akyüz C, Kutluk T and Büyükpamukçu M: Langerhans cell histiocytosis: retrospective analysis of 217 cases in a single center. Pediatr Hematol Oncol 25: 399-408, 2008.

2. Lau SK, Chu PG and Weiss LM: Immunohistochemical expression of langerin in Langerhans cell histiocytosis and non-Langerhans cell histiocytic disorders. Am J Surg Pathol 32: 615-619, 2008

3. Abla O, Egeler RM and Weitzman S: Langerhans cell histiocytosis: current concepts and treatments. Cancer Treat Rev 36: 354-359, 2010.

4. Stockschlaeder M and Sucker C: Adult Langerhans cell histiocytosis. Eur J Haematol 76: 363-368, 2006.

5. Satter EK and High WA: Langerhans cell histiocytosis: a review of the current recommendations of the Histiocyte Society. Pediatr Dermatol 25: 291-295, 2008.

6. Weismüller TJ, Wedemeyer J, Kubicka S, Strassburg CP and Manns MP: The challenges in primary sclerosing cholangitisaetiopathogenesis, autoimmunity, management and malignancy. J Hepatol 48: S38-S57, 2008

7. Wong A, Ortiz-Neira CL, Reslan WA, Sharon R, Pinto-Rojas A, Kaura D and Anderson R: Liver involvement in Langerhans cell histiocytosis. Pediatr Radiol 36: 1105-1107, 2006.

8. Schmidt S, Eich G, Hanquinet S, Tschäppeler H, Waibel P and Gudinchet F: Extra-osseous involvement of Langerhans' cell histiocytosis in children. Pediatr Radiol 34: 313-321, 2004.

9. Windebank K and Nanduri V: Langerhans cell histiocytosis. Arch Dis Child 94: 904-908, 2009.

10. Histocyte Society 2009. Langerhans cell histiocytosis evaluation and treatment guidelines. Histocyte Society online, 2009, cited 2010-02. Available from: http://www.histiocytesociety.org/site/c. $\mathrm{mqISL} 2 \mathrm{PIJrH} / \mathrm{b} .4442715 / \mathrm{k}$.A339/Treatment_Plans.htm.

11. Henter JI, Tondini C and Pritchard J: Histiocyte disorders. Crit Rev Oncol Hematol 50: 157, 2004. 